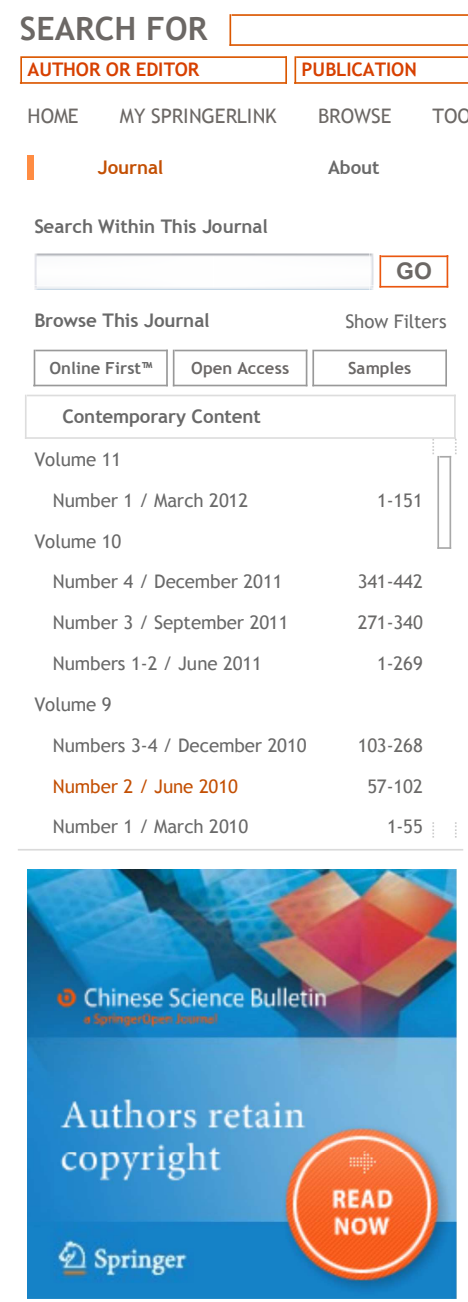

Volume 9, Number 2 / June 2010

Viewing all 5 articles verification by LDMOS
Journal of Computational Electronics

Volume 1 / 2002 - Volume 11 / 2012
You have Guest access.

What can I do as a guest? LOG IN

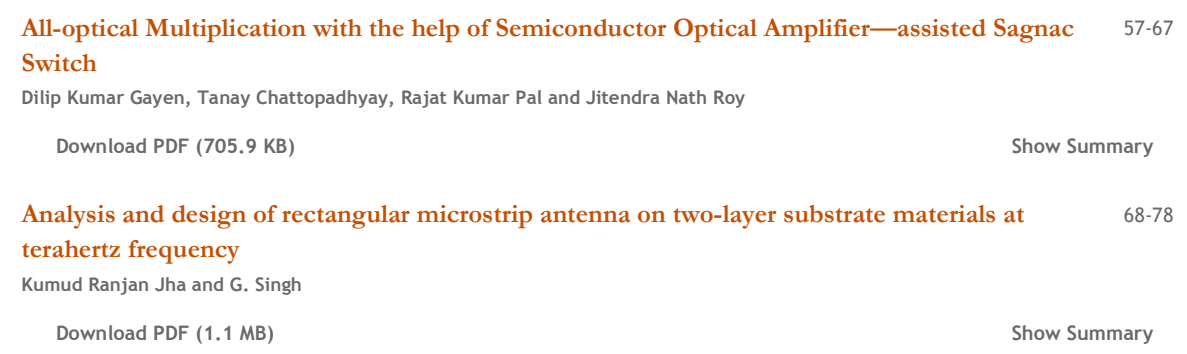

All-optical Multiplication with the help of Semiconductor Optical Amplifier-assisted Sagnac 57-67 Switch

Dilip Kumar Gayen, Tanay Chattopadhyay, Rajat Kumar Pal and Jitendra Nath Roy Download PDF (705.9 KB)

Show Summary

Analysis and design of rectangular microstrip antenna on two-layer substrate materials at 68-78 terahertz frequency

Kumud Ranjan Jha and G. Singh

Download PDF (1.1 MB)

Show Summary

A TCAD approach for non-linear evaluation of microwave power transistor and its experimental 79-86

Ahsan Ullah Kashif, Christer Svensson, Khizar Hayat, Sher Azam and Nauman Akhter, et al.

Download PDF (595.5 KB) Show Summary

Performance optimization of MOS-like carbon nanotube-FETs based on electrostatic doping 87-92 Hailiang Zhou, Minxuan Zhang and Yue Hao

Download PDF (432.2 KB) Show Summary

Computational modeling of stochastic processes in electron amplifiers $\quad 93-102$ Alla Shymanska

Download PDF (413.8 KB) _ Show Summary

\title{
Engineering Design
}

Structural, Civil, Mechanical - Geotech, drainage, subdivisions.

www. burrett.co.nz

\section{AdChoices D}

Share this Item

email citeulike Connotea Delicious

FAQ General info on journals and books Send us your feedback Impressum Site Map Contact us

(c) Springer, Part of Springer Science+Business Media Privacy, Disclaimer, Terms \& Conditions, and Copyright Info 